\title{
Study of erythrocytes as a novel drug carrier for the delivery of artemether
}

\author{
Muhammad Ijaz Riaz', Hafiz Shoaib Sarwar, ${ }^{1,2}$, Mubashir Rehman ${ }^{3}$, Umar Farooq Gohar,, , Syed \\ Atif Raza ${ }^{4}$, Muhammad Irfan Siddique ${ }^{5}$, Gul Shahnaz ${ }^{2 *}$,Muhammad Farhan Sohail ${ }^{1,2 *}$
}

\begin{abstract}
${ }^{1}$ Riphah Institute of Pharmaceutical Sciences, Riphah International University Lahore Campus, Lahore, Pakistan, ${ }^{2}$ Department of Pharmacy, Faculty of Biological Sciences, Quaid-i-Azam University, Islamabad, Pakistan, ${ }^{3}$ Faculty of Pharmacy, University of Central Punjab, Lahore, Pakistan, ${ }^{4}$ University College of Pharmacy, University of the Punjab, Allama Iqbal Campus, Lahore, Pakistan, ${ }^{5}$ Institute of Pharmaceutical Sciences, University of Veterinary and Animal Sciences, Lahore, Pakistan, ${ }^{6}$ Department of Industrial Biotechnology, Government College University, Lahore, Pakistan
\end{abstract}

\begin{abstract}
Resealed erythrocytes have been explored in various dimensions of drug delivery, owing to their high biocompatibility and inability to initiate immune response. The present research was designed to evaluate the drug delivery potential of erythrocytes by loading a hydrophobic anti-malarial drug, Artemether. Three different loading techniques were applied to achieve maximum optimized drug loading. A HPLC method was validated for drug quantification in erythrocytes. The relatively high loading was achieved using hypotonic treatment was $31.39 \%$ as compared to other two methods. These, drug loaded erythrocytes were characterized for membrane integrity via ESR showing higher ESR values for drug loaded cells as compared to normal cells. Moreover, microscopic evaluation was done to observe morphological changes in erythrocytes after successful loading which showed swollen cells with slight rough surface as compared to smooth surface of normal cells. Drug release was studied for $8 \mathrm{~h}$ which showed more than $80 \%$ release within 3-7 h from erythrocytes treated with different hypotonic methods. Overall, the study revealed a potential application of erythrocytes in delivery of hydrophobic drugs using hypotonic treatment as compared to other methods.
\end{abstract}

Keywords: Resealed Erythrocytes. Novel Drug Delivery System. Hydophobic drug/artemether.

\section{INTRODUCTION}

According to the world health organization (WHO) malaria occupies the first place in parasitic diseases that can kill the people worldwide. An estimated 500 million cases are reported annually with 1-2.5 million deaths out of which majority of children under five years of age (Organization, 2015). It is caused by the parasites of genus Plasmodium belonging to species Plasmodium vivax, P. ovale, P. malariae, P. falciparum. While taking the blood meal the female anopheles mosquito transmits the sporozoites of plasmodium parasites into the blood of the host. Sporozoites reach the blood within short period

\footnotetext{
*Correspondence: M. F. Sohail. Riphah Institute of Pharmaceutical Sciences, Riphah International University Lahore Campus, Lahore, Pakistan. Phone: +92 344 4120001. E-mail: farmacist.pk@gmail.com

G. Shahnaz. Department of Pharmacy, Faculty of Biological Sciences, Quaidi-Azam University, Islamabad, Pakistan. Phone: +92 51 90644137. E-mail: gshahnaz@qau.edu.pk (iD)
}

of time Within a short period of time the sporozoites reaches the blood and are transported to the liver where they converted to merozoites, enter blood circulation and invade RBCs. Within RBCs these are multiplied via asexual reproduction and infect other RBCs, thus disseminating the infection (Matteelli et al., 1997).

Currently, there is no approved malaria vaccine available in market and as the currently available RTS,S/ AS01 vaccine has shown less than $50 \%$ efficacy, thus, is not recommended by WHO (Gosling et al., 2016). Therefore, the treatment of malaria relies mainly on the use of chemotherapeutic agents. Quinine was the first drug used to treat malaria which later became less effective leading to the discovery and development of new antimalarial drugs. In 1940 s, a German scientist synthesized chloroquine, an effective alternative of quinine which showed remarkable effectiveness during World War II (Renslo, 2013). High therapeutic doses of chloroquine are required because of greater trapping into 
kidneys, liver, lung and spleen. In turn, use in higher doses for about 3-6 years may results in renal toxicity. In turn, the higher doses may result in toxicity such as retinal toxicity after using for 3-6 years (L'Estrange, 1987). A number of new molecules have been developed to treat malaria better than quinine and chloroquine. Artemether (ARM) is one of those anti-malarial drugs that inhibits $P$. falciparum encoded sarcoplasmic-endoplasmic reticulum calcium ATPase and clear parasitaemias better than chloroquine (Flannery, Chatterjee, Winzeler, 2013). Artemether is also reported to reduce gametocytogenesis by acting on the ring stages in early stages of gametocytes. Artemether is mainly metabolized by CYP450 2B6, CYP450 3A4 and possibly CYP450 2A6. The drug is extensively and rapidly metabolized into dihydroartemisinic that is the main active metabolite. However, a very short half-life, lack of oral absorption and poor aqueous solubility limits is use (Karbwang et al., 1997). Currently, an oil-based injectable formulation for intramuscular administration is available commercially. However, its use is limited due to its non-defined and erratic absorption. So, there is need of developing a suitable drug delivery system for the intravenous delivery of ARM (Bhadra, Bhadra, Jain, 2005).

Novel drug delivery systems (NDDS) are amongst the widely explored delivery systems to overcome various limitations faced by the conventional drug delivery systems. Various NDDS including liposomes, niosomes, polymeric and metallic nanoparticles, dendrimers and many others are reported with some good potential in drug delivery. Some of these NDDS have been approved by FDA and a lot more are in process of approval (Hassan et al., 2017). In this scenario, drug loaded erythrocytes or resealed erythrocytes have been reported as a promising delivery system for drugs and various enzymes (Tyrrell, Ryman, 1976). The most important benefits they present is the biocompatibility and biodegradation with long circulating half-life and can be loaded with numerous biological agents. Resealed erythrocytes can easily be prepared by obtaining blood from various organisms and erythrocytes are separated using many techniques. These erythrocytes are then treated with different processes to load various biological agents and injecting back to the same organism. Upon injection of the drug loaded erythrocytes, they act as slow drug releasing depot and target reticulo-endothelial system (RES) and prevent the enzymatic degradation (Gothoskar, 2004). To control the dissemination of malaria the drug delivery via RBCs can be of promising strategy as the newly released merozoites will also attack the drug loaded RBCs along with normal RBCs where they encounter the high concentration of therapeutic agents, thus providing a suitable control of malarial infection.

This study was designed to develop and in vitro characterize ARM loaded erythrocytes as an efficient carrier. Hypotonic treatment is reported as an efficient method for drug loading with less effects on RBCs. Three different techniques based on hypotonic treatment were applied to check the effect on loading efficiency, ESR and drug release from RBCs to develop an optimized delivery system.

\section{MATERIAL AND METHODS}

\section{Material}

Sodium chloride, Giemsa, sodium hydroxide and potassium dihydrogen phosphate were purchased from Sigma-Aldrich (Germany). Water, methanol and acetonitrile were of HPLC grade. Artemether was received as a gift from NovaMed Pharmaceuticals (Pvt.) Ltd, Lahore Pakistan.

\section{Methods}

\section{Erythrocytes isolation}

Fresh human blood was withdrawn via vein puncture with volunteer's consent and stored at $4^{\circ} \mathrm{C}$ using the reported method (Sohail et al., 2017). The erythrocytes were separated from blood by centrifugation at $1000 \mathrm{rpm}$ for $20 \mathrm{~min}$. The pellet containing RBCs was collected and washed with phosphate buffer solution ( $\mathrm{pH}$ 7.4). These isolated RBCs were used for the preparation of resealed erythrocytes using different methods of drug loading.

\section{Drug loading techniques}

Three different drug loading methods were applied to study the effect of process variables on achieving maximum loading of ARM along with RBCs membrane integrity to control the drug release from loaded RBCs. The detailed procedures are described below.

\section{Method A (Hypotonic dilution)}

A volume of $8 \mathrm{~mL}$ of washed erythrocytes was taken in the test tube and $10 \mathrm{~mL}$ of the $4 \%$ ARM solution in acetonitrile and water was added. The tonicity of the drug solution was adjusted with hypotonic phosphate buffer to $\mathrm{pH}$ 7.4. For $20 \mathrm{~min}$, the resultant mixture was centrifuged at $1000 \mathrm{rpm}$. The supernatant was removed and discarded. The pellet was washed with phosphate buffer solution. The pellet containing loaded erythrocytes was stored in refrigerator until further characterization (Ihler, Tsang, 1987). 


\section{Method B (Hypotonic pre-swelling)}

In method $\mathrm{B}$, a hypotonic solution of $\mathrm{NaCl}(0.3 \%$ $\mathrm{w} / \mathrm{v})$ was added to flask with erythrocytes $(50 \% \mathrm{v} / \mathrm{v})$. The RBCs were allowed to swell and $10 \mathrm{~mL}$ of drug solution $(4 \% \mathrm{w} / \mathrm{v})$ was then added and sonicated to facilitate the drug encapsulation into erythrocytes. The isotonicity of swelled RBCs was retained by adding $1.3 \% \mathrm{w} / \mathrm{v} \mathrm{NaCl}$ solution and incubated at $0^{\circ} \mathrm{C}$ for $5 \mathrm{~min}$. Then sample was centrifuged to remove the un-encapsulated drug. The supernatant was removed and discarded. The suspension of RBCs was washed three times with phosphate buffer solution at $\mathrm{pH} 7.4$ to remove any free drug and diluted suitably with the same buffer solution. The final preparation was stored at $4^{\circ} \mathrm{C}$ in refrigerator until further characterization (Alpar, Irwin, 1987).

\section{Method C (Modified hypotonic pre-swelling)}

In method $\mathrm{C}$, a hypotonic solution containing $\mathrm{NaCl}$ $(0.6 \% \mathrm{w} / \mathrm{v})$ was added to flask with erythrocytes $(50 \%$ $\mathrm{v} / \mathrm{v})$. The RBCs were allowed to swell and $10 \mathrm{~mL}$ of drug solution $(4 \% \mathrm{w} / \mathrm{v})$ was added and sonicated to facilitate the drug encapsulation into erythrocytes. The isotonicity of swelled RBCs was retained by adding $\mathrm{NaCl}$ solution $(1 \% \mathrm{w} / \mathrm{v})$ and incubated at $0^{\circ} \mathrm{C}$ for $5 \mathrm{~min}$ followed by centrifugation to remove the un- encapsulated drug. The suspension of RBCs was washed three times with phosphate buffer solution at $\mathrm{pH} 7.4$ and diluted suitably with the same buffer solution. The final preparation was stored at $4^{\circ} \mathrm{C}$ in refrigerator until further characterization (Pitt et al., 1983).

\section{Characterization of drug loaded erythrocytes}

\section{Validation of HPLC Method Used for ARM quantification}

The HPLC method was validated using International council for Harmonisation ( $\mathrm{ICH}$ ) guidelines for ARM quantification in different experiments. Mobile phase consisting of acetonitrile and deionized water was prepared in ratio of 93:7, respectively. The $\mathrm{pH}$ of the mobile phase was adjusted with dilute sulphuric acid up to 3.0. The mobile phase was used as diluent as well. $4 \mathrm{mg} / \mathrm{mL}$ of ARM solution was used in HPLC (Waters e2695) as reference standard. Nominally $4 \mathrm{mg} /$ $\mathrm{mL}$ of ARM obtained from drug loaded erythrocytes in method A, method B and method C was used as sample solution. Column having specifications $250-\mathrm{cm} \times 4.6-$ $\mathrm{mm}$; 5- $\mu \mathrm{m}$ packing $\mathrm{L} 1$ was used at ambient temperature and UV detector was set at $216 \mathrm{~nm}$ wave length in HPLC. The $20 \mu \mathrm{L}$ injection volume was run at the flow rate of $1.0 \mathrm{~mL} / \mathrm{min}$.

\section{Drug loading efficiency}

Drug loading efficiency in RBCs was estimated through a method reported earlier (Hirlekar et al., 2008). Briefly, $1 \mathrm{~mL}$ of drug loaded erythrocytes was centrifuged and the pellet containing loaded erythrocytes was taken for drug quantification. The pellet was resuspended in $1 \mathrm{~mL}$ of distilled water and heated for $20 \mathrm{~min}$ at $40^{\circ} \mathrm{C}$ followed by filtration through $0.22 \mu \mathrm{m}$ filter. The Drug contents in filtrate were estimated by the HPLC method reported earlier. The drug was estimated by using the following equation.

$\%$ age content of drug $=\frac{\text { Area of sample } \times \text { weight of standard } \times 5}{\text { Area of standard } \times 25 \times \text { weight of sample }} \times 100$

The same method was applied for quantification of drug loading in RBCs from other two methods.

\section{Erythrocytes membrane integrity analysis}

To distinguish between loaded and unloaded red blood cells and effect of different loading techniques on erythrocyte membrane integrity, erythrocytes sedimentation rate (ESR) was performed (Saulis, 2005). Briefly, drug loaded, and normal erythrocytes were washed with buffer solution and were drawn in the WESTERGREN tube up to 0 mark with the help of rubber bulb and wiped out the bottom of the tube with cotton. The tube was set snugly upright on the WESTREGREN stand and made sure that there was no leakage in vertical position. The tube was left undisturbed for $1 \mathrm{hr}$ and after $1 \mathrm{hr}$ read the results of sedimentation of the erythrocytes. The distance covered by erythrocytes during fall as well as the height of clear plasma (in millimeters) at the top of the column were measured for the further calculations.

\section{Microscopic morphology studies of erythrocytes}

The morphology of drug loaded, and blank erythrocyte was studied via optical microscopy. The erythrocytes were washed with double distilled water and $10-15 \mu \mathrm{L}$ blood was applied on glass slide to make a uniform smear. The smear was air dried and fixed with methanol (99.8\%) solution and air dried again. The slides were stained with the commercially available Giemsa stain (1:9, Giemsa: distilled water). The staining was carried out for $15 \mathrm{~min}$. After that, the slides were washed with the water and air dried. The margins of the slides were cleaned with the alcoholic swab. The slides were observed under microscope to observe the morphology of erythrocytes before and after different treatments. 


\section{Drug release profile}

Drug release from RBCs was estimated using membrane dialysis method as reported earlier (Sohail et al., 2016). Briefly, measured volume of ARM loaded $\mathrm{RBCs}$ was diluted in $5 \mathrm{~mL}$ of PBS $(\mathrm{pH}=7.4)$ and enclosed in dialysis membrane with pore size $12 \mathrm{KDa}$. The dialysis bag was immersed in $25 \mathrm{~mL}$ PBS having $1 \%$ tween 80 to maintain the sink condition. The system was maintained at $37^{\circ} \mathrm{C}$ with $50 \mathrm{rpm}$. The samples were taken at pre-defined time intervals and replaced with fresh media. The samples were analyzed using HPLC method to estimate release profile from RBCs. Same method was repeated for all the three loading techniques.

\section{RESULTS AND DISCUSSION}

\section{Erythrocytes isolation}

Resealed erythrocytes have shown good potential to be developed as novel drug delivery system owing to their numerous advantages. High intracellular volume of erythrocytes enables the loading of high drug contents and provides good control over drug release. ARM was successfully loaded in fresh human erythrocytes and characterized for various parameters. Erythrocytes were successfully isolated from fresh human blood via centrifugation at $1000 \mathrm{rpm}$ for $20 \mathrm{~min}$. The washed erythrocyte pallet was suspended in isotonic buffer solution and stored in refrigerator till further use in different studies. The isolated RBCs appeared regular biconcave shape showing now effect on structure during isolation

\section{HPLC method validation}

HPLC method was successfully validated for ARM quantification in erythrocytes following ICH guidelines on six basic attributes i.e. precision, linearity, range,

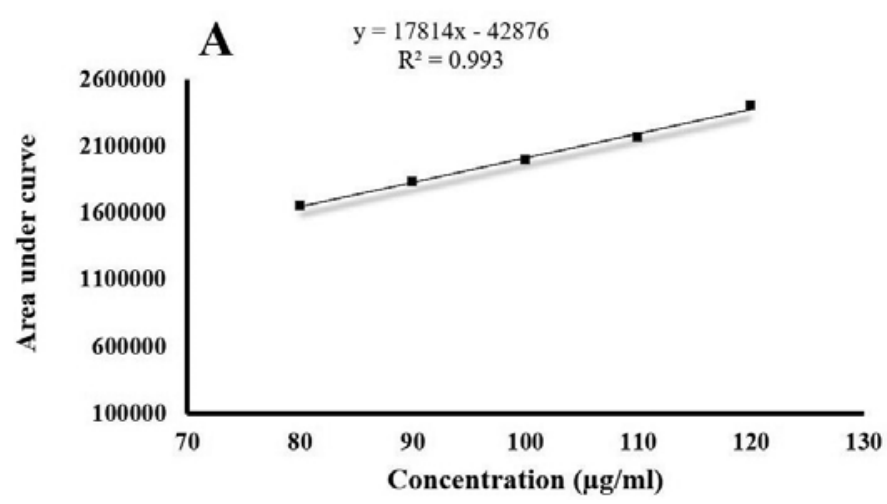

specificity, accuracy, robustness. The results determined that all the parameters of instrument precision were well within the specified limits that ensured the validity of the developed method. The linearity of the data over a range of 80 to $120 \mu \mathrm{g} / \mathrm{mL}$ was observed and a calibration curve was drawn with coefficient of correlation $\left(\mathrm{R}^{2}\right)$ 0.993. This indicated that the test procedure followed the Beer's law. The results of instrument specificity indicated the specificity obtained for ARM and placebo which confirmed that only standard solution gave peak of ARM at retention time of $5.123 \mathrm{~min}$ as shown in Figure 1 whereas, no peak was observed for placebo. The method showed good repeatability in terms of inter-day and intra-day variations confirming the precision of results.

\section{Drug loading}

Hypotonic treatment for drug loading is reported for various molecules. Three different modified hypotonic treatments were used to load ARM in RBCs. All the three methods were quite successful in loading ARM into erythrocytes with varying loading concentrations as shown in Figure 2A. These were quantified via HPLC methods validated for ARM. Method A was based on hypertonic treatment of erythrocytes resulting in shrinkage of cells followed by isotonic treatment. Method B and C were based on hypotonic treatment with varying concentration of $\mathrm{NaCl}$ solutions resulting in swelling of erythrocytes enabling drug loading into the cells. ARM loading was quantified using the developed HPLC method and was found to be $11.64 \%, 14.56 \%$ and $31.39 \%$ with method $\mathrm{A}$, method $\mathrm{B}$ and method $\mathrm{C}$ respectively. Method A resulted in lowest loading efficiency as the drug crossed the cell membrane by diffusion method, following the concentration gradient and the number of pores produced in cell membranes was less in number to take drug inside the cells. Also, the loaded cells were incubated

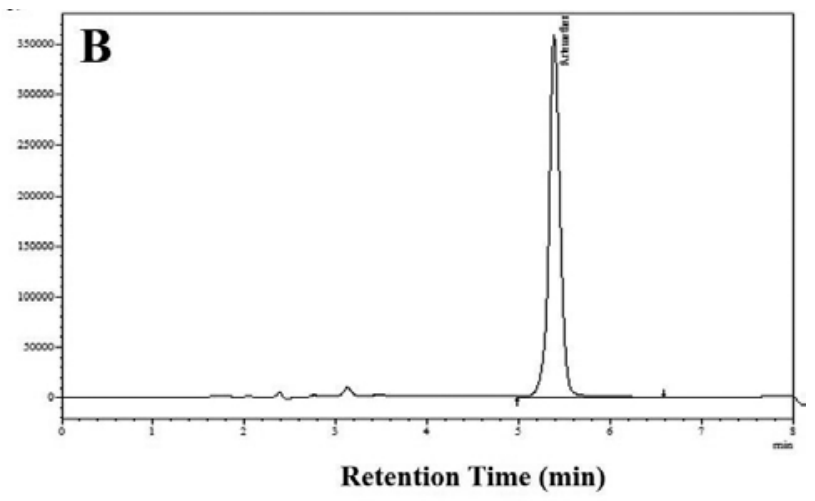

FIGURE 1 - (A) calibration curve of HPLC method showing linearity over a range of 80-120 $\mu \mathrm{g} / \mathrm{mL}$, (B) HPLC standard chromatogram showing retention peak of artemether at $5.123 \mathrm{~min}$. 
at $40^{\circ} \mathrm{C}$ for drug analysis, the drug might be degraded, and chromatograph results showed low amount of drug encapsulation.

The lower loading efficiency with method B was again resulted because of the incubation of the cells at $0-4^{\circ} \mathrm{C}$ which solidified the cell membrane fatty acids and resisted the cellular lysis during drug loading and prior to the treatment of erythrocytes with hypotonic solution. The hypotonic salt solution produced low number of pores in cell membrane because of swelling of the cells due to hydrostatic pressure inside the cells which was created by the osmotic movement of the water molecules across the membrane. Those pores facilitated the entry of drug molecules inside the cells and enhanced the drug encapsulation efficiency that was $14.56 \%$. The other possible reason might be the lysis of the cells in $0.3 \%$ $\mathrm{NaCl}$ solution and incubation at $40^{\circ} \mathrm{C}$ for drug analysis which affected the drug stability and premature release from erythrocyte destruction. Method $\mathrm{C}$ resulted in highest drug loading of $31.39 \%$. This might attribute a number of factors like the increased refrigeration of erythrocytes at $0-4^{\circ} \mathrm{C}$, prior to hypotonic treatment, resulted in more solidification of the cell membrane components thus prevented the cellular lysis. The loading of ARM drug into erythrocytes was low as compared to ARM following same method $\mathrm{C}$, this might be due to higher molecular weight and differences in other parameters such as incubation period and incubation temperature of erythrocytes. Moreover, the cell lysis in method $\mathrm{B}$ and $\mathrm{C}$ was prevented by treating the cells with $0.6 \% \mathrm{NaCl}$ solution. Which caused opening of cellular pores and less cellular lysis.

\section{ESR analysis}

Erythrocyte sedimentation rate (ESR) is a simple and inexpensive laboratory test to access acute phase response.

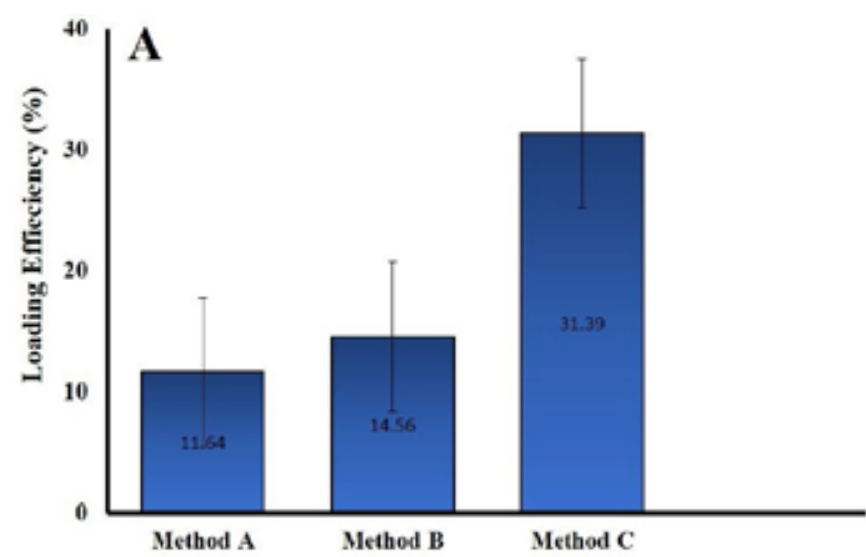

It is the estimation of suspending stability of RBCs and is relate to number and size of RBCs and to relative concentration of plasma proteins. The effect of three treatments on RBCs membrane integrity was estimated via erythrocyte sedimentation rate (ESR) analysis. The sedimentation rate for loaded and unloaded erythrocytes have been shown in Figure 2B. The result showed normal ESR of untreated RBCs as compared to drug loaded RBCs. The values of ESR for drug loaded erythrocytes were observed to be $51 \mathrm{~mm} / \mathrm{h}, 63 \mathrm{~mm} / \mathrm{h}$ and $90 \mathrm{~mm} / \mathrm{h}$ for method A, method B and Method C respectively. The ESR value of drug loaded erythrocytes was higher than normal ESR value $(0-15 \mathrm{~mm} / \mathrm{h})$ erythrocytes because of increase in the mass of red blood cells due to drug loading. As the maximum drug loading into erythrocytes was achieved in method C that's why the ESR value in the erythrocytes of method $\mathrm{C}$ was greater than all the other erythrocytes obtained from all other drug loading methods.

\section{Drug release studies}

Once successfully encapsulated, the drug must come out of the carrier to produce desired effect. A number of mechanism are suggested by which drug is released from resealed erythrocytes. Post hypotonic treatment may result in decreasing membrane integrity because of pore formation (Pribush, Meyerstein, Meyerstein, 2002). During circulation, they are readily taken up by RES and other stress mechanisms which degrade them to release drug. Drug release from ARM loaded RBCs was evaluated for 8 hours using PBS having $\mathrm{pH}=7.4$ as medium via membrane dialysis method. The results obtained are shown in Figure 3 indicating percentage drug release at various time intervals. RBCs produced from method $\mathrm{C}$ showed maximum release (above $85 \%$ ) within $4 \mathrm{~h}$ as compared to Method B which showed around $80 \%$ release

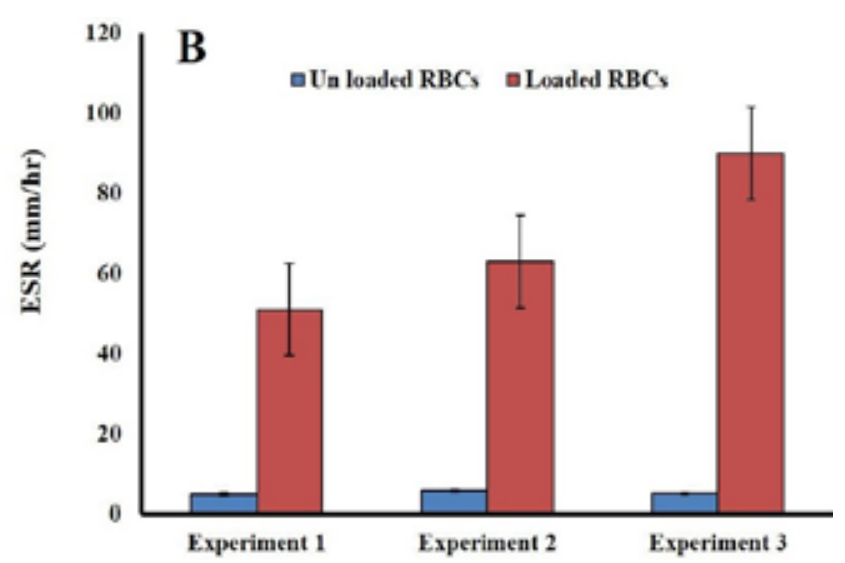

FIGURE 2 - (A) Drug loading efficiency of 3 different method used for erythrocytes. (B) effect of different loading techniques on ESR showing integrity of membrane. The results are presented as Mean \pm SD of three experiments. 
in same time. Method A showed better release profile as only $65 \%$ of the encapsulated drug was released in $4 \mathrm{~h}$. Furthermore, these RBCs showed gradual release of ARM from the loaded cells as the treatment followed by method A might have caused less damage to the cell membrane and drug retained for longer period of time with survival of cells for longer period. Also, the possible reason behind less control on drug release by method $\mathrm{B}$ and $\mathrm{C}$ suggests the involvement of membrane integrity and amount of drug loaded inside the RBCs. The higher loaded amount along with high treatment variables affected the membrane integrity more as compared to low drug loading.

\section{Microscopic evaluation of cell morphology}

The change in erythrocyte morphology after successful drug encapsulation was assessed by microscopic examination of stained erythrocytes. Both drug loaded
(Method A) and normal erythrocytes were examined at $100 \mathrm{x}$ resolution to observe the differences and are shown in Figure 4a \& 4b. The drug loaded cell appeared much increased in size as compared to normal cells when observed at same magnification. The hollow central compartment seen in normal cells, was disappeared in drug loaded cells, suggesting the encapsulation of drug in intracellular space. Moreover, the membrane appeared a bit rough and irregular shape in appearance after hypotonic treatment compared to the smooth and spherical appearance of erythrocytes in normal sample.

\section{CONCLUSIONS}

Drug loaded erythrocytes are the safe and effective for sustained drug delivery as this novel system has is biocompatible. Moreover, targeting to the organ of the reticule-endothelial system is possible with loaded

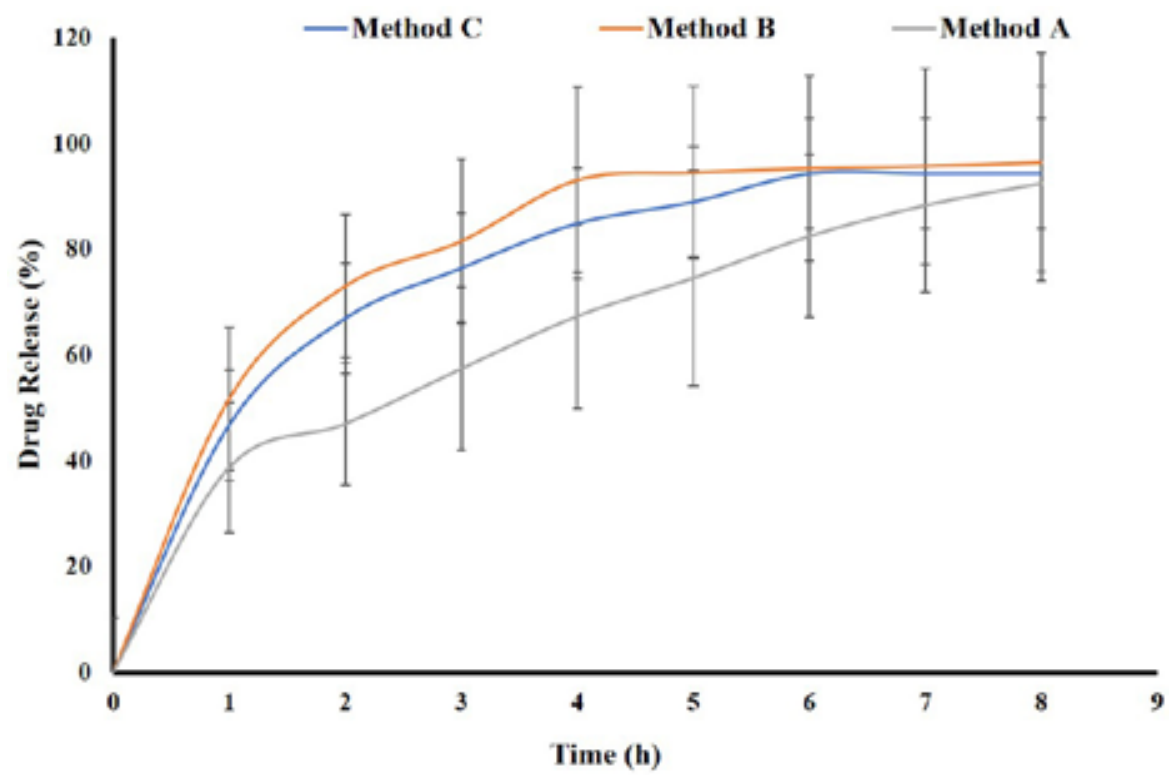

FIGURE 3 - In-vitro release of artemether from erythrocytes followed by three different loading techniques. The results are presented as Mean \pm SD of three experiments.
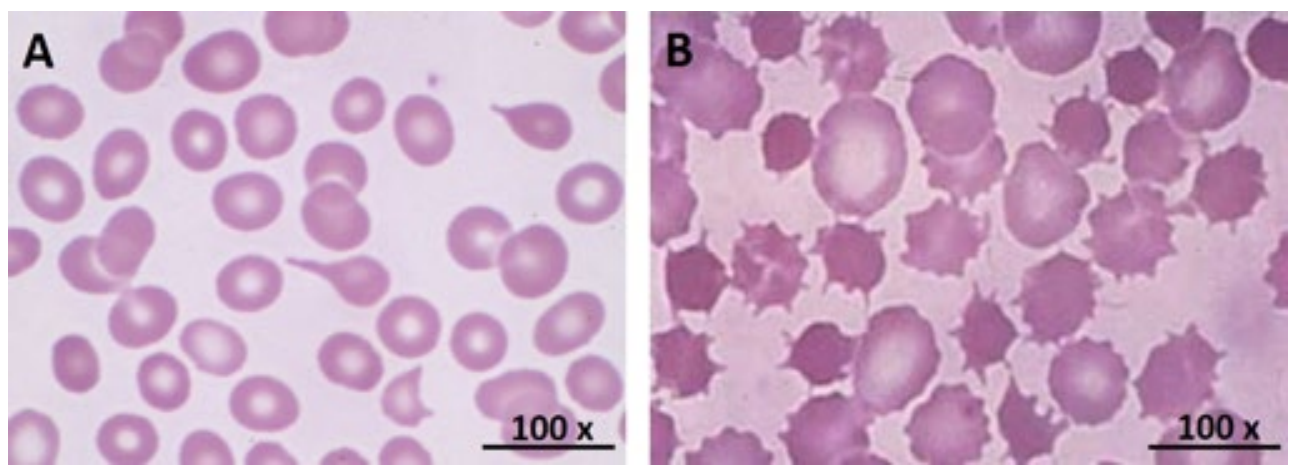

FIGURE 4 - Giemsa stained RBCs (A) Normal (B) Drug loaded showing changes in morphology before and after drug loading techniques. 
erythrocytes. The loading of antimalarial drug in erythrocytes will follow the targeted missile technology for plasmodium present in blood stream. The lipophilic drugs cannot be administered intravenously, due to requirement of large volume of vehicle, but this erythrocyte based drug delivery system will make it quite possible. The hypotonic treatment showed good drug loading for lipophilic drugs with an improved membrane integrity and drug release over a longer period of time. This could be helpful in designing relatively more stable formulation. Overall, the study suggested that these RBCs possess potential to be taken up further in designing an efficient drug delivery system for lipophilic drug molecules.

\section{ACKNOWLEDGMENT}

We are extremely grateful to NovaMed Pharmaceuticals (Pvt.) Ltd, Lahore Pakistan for facilitating the completion of study.

\section{AUTHOR'S DECLARATION}

The authors declare no conflict of interest.

\section{REFERENCES}

Alpar H, Irwin W. Some unique applications of erythrocytes as carrier systems. Adv Biosci. 1987;67:1-9.

Bhadra D, Bhadra S, Jain N. Pegylated lysine based copolymeric dendritic micelles for solubilization and delivery of artemether. J Pharm Pharm Sci. 2005;8(3):467-482.

Flannery EL, Chatterjee AK, Winzeler EA. Antimalarial drug discovery-approaches and progress towards new medicines. Nat Rev Microbiol. 2013;11(12):849-62.

Gosling R, von Seidlein L. The future of the RTS, S/AS01 malaria vaccine: an alternative development plan. PLoS Med. 2016;13(4):e1001994.

Gothoskar AV. Resealed erythrocytes: a review. Pharm Technol. 2004;28(3):140-155.

Hassan S, Prakash G, Ozturk AB, Saghazadeh S, Sohail MF, Seo J. et al. Evolution and clinical translation of drug delivery nanomaterials. Nano Today. 2017;15:91-106.

Hirlekar R, Patel P, Dand N, Kadam V. Drug loaded erythrocytes: as novel drug delivery system. Curr Pharm Design. 2008;14(1):63-70.
Ihler GM, Tsang HC-W. [21] Hypotonic hemolysis methods for entrapment of agents in resealed erythrocytes Methods in enzymology. v. 149, p. 221-229. Netherlands: Elsevier; 1987.

Karbwang J, Na-Bangchang K, Congpuong K, Molunto $\mathrm{P}$, Thanavibul A. Pharmacokinetics and bioavailability of oral and intramuscular artemether. Eur J Clin Pharmacol. 1997;52(4):307-310.

L'Estrange OM. Side effects of quinine and derivatives. Acta Leidensia. 1987;55:77-86.

Matteelli A, Castelli F, Caligaris S. Life cycle of malaria parasites. Handbook of Malaria infection in the tropics. 1st edn. Bologna: Associazione Italiana "Amici di R. Follereau"; 1997. p. 17-23.

Pitt E, Johnson CM, Lewis DA, Jenner DA, Offord RE. Encapsulation of drugs in intact erythrocytes: an intravenous delivery system. Biochem Pharmacol. 1983;32(22):3359-3368.

Pribush A, Meyerstein D, Meyerstein N. Kinetics of erythrocyte swelling and membrane hole formation in hypotonic media. Biochim Biophys Acta (BBA) Biomembr. 2002;1558(2):119132.

Renslo AR. Antimalarial drug discovery: from quinine to the dream of eradication. Washington: ACS Publications; 2013.

Saulis G. The loading of human erythrocytes with small molecules by electroporation. Cell Mol Biol Lett. 2005;10(1):2335.

Sohail MF, Javed I, Hussain SZ, Sarwar S, Akhtar S, Nadhman A, et al. Folate grafted thiolated chitosan enveloped nanoliposomes with enhanced oral bioavailability and anticancer activity of docetaxel. J Mat Chem B. 2016;4(37):6240-6248.

Sohail MF, Sarwar HS, Javed I, Nadhman A, Hussain SZ, Saeed $\mathrm{H}$, et al. Cell to rodent: toxicological profiling of folate grafted thiomer enveloped nanoliposomes. Toxicol Res. 2017;6:814-821.

Tyrrell DA, Ryman BE. The entrapment of therapeutic agents in resealed erythrocyte 'ghosts' and their fate in vivo. London: Portland Press Limited; 1976.

World Health Organization. WHO. World Health Statistics 2015. Genva: World Health Organization; 2015.

Received for publication on $22^{\text {nd }}$ October 2017 Accepted for publication on $27^{\text {th }}$ April 2018 